\title{
The Dual-axes for Soft X-Ray Cryo-tomography Reveals Ultrastructural Alterations of the Host Cell during Hepatitis C Infection by Increasing the Isotropic Axial Resolution
}

Perez-Berna $\mathrm{AJ}^{1}$, Valcarcel $\mathrm{R}^{1}$, Rodríguez $\mathrm{MJ}^{2}$, Chichon $\mathrm{FJ}^{2}$, Sorrentino ${ }^{1}$, Carrascosa $\mathrm{JL}^{2}$, Gastaminza $\mathrm{P}^{2}$ and Pereiro $\mathrm{E}^{1}$

1. MISTRAL Beamline, ALBA-CELLS synchrotron , Barcelona, España

2. Centro Nacional de Biotecnología, Consejo Superior de Investigaciones Científicas, Madrid, España

Soft X-Ray cryo tomography has become a tool for analyzing the ultrastructure of intact and cryoprepared cells. No other method can provide better 3D resolution of the whole cell organelles preserved close to native state. Nevertheless the tilt angle range from which data can be gathered is limited from $65^{\circ}$ to $65^{\circ}$ or $-70^{\circ}$ to $70^{\circ}$ depending on the zone plate used. This limitation causes the truncation of the data, represented in reciprocal space as a missing wedge which is an important artifact in the reconstructed tomograms that affects the axial direction. The effect of the missing wedge is very strong on details perpendicular to the optical axis. One way to reduce this artefact is to acquire data from two perpendicular tilt axes, a technique called "dual axis tomography." I In the current work we have designed a holder for MISTRAL microscope that facilitate the acquisition of two tilt series of the same area of interest whose tilt axes are mutually perpendicular. Dual Tilt leaded to decrease the missing wedge to a missing pyramid and to obtain a more isotropic axial resolution in the volume helping to visualized structures that were hidden in the single tilt volume due to their orientation with respect to the axis of rotation. The dual axis tilting method increases the visibility of certain features which are hidden by the missing wedge; this makes the resolution achievable more isotropic inside the volume (Fig 1). On the other hand the new holder allows us loading samples in an autogrid ring. The autogrid system protects grids during handling and facilitates the correlative microscopy making possible the analysis of the same samples with different techniques. Correlation of cryo-fluorescence microscopy and cryo-SXT can be used to localize fluorescent proteins tagging specific organelles. Cryo-correlative light and X-ray microscopy (cryo-CLXM) is particularly useful for the study of organelles that are susceptible to chemical fixation artifacts during sample preparation for electron microscopy ${ }^{2}$. We have selected the Hepatitis $\mathrm{C}$ virus as a biological model for the study.

Chronic hepatitis $\mathrm{C}$ virus (HCV) infection causes severe liver disease in millions of humans worldwide. Pathogenesis of HCV infection is strongly driven by a deficient immune response of the host, although intersection of different aspects of the virus life cycle with cellular homeostasis is emerging as an important player in the pathogenesis and progression of the disease.

Cryo soft X-ray tomography (cryo-SXT) was performed to investigate the ultrastructural alterations induced by the interference of hepatitis $\mathrm{C}$ virus (HCV) replication with cellular homeostasis. Native, whole cell, three-dimensional maps were obtained in HCV replicon-harboring cells and in a surrogate model of HCV infection at 40nm resolution. Tomograms from HCV-replicating cells show blind-ended endoplasmic reticulum (ER) tubules with pseudo spherical extrusions and marked alterations of mitochondrial morphology that correlated topologically with the presence of ER alterations, suggesting a short-range influence of the viral machinery on mitochondrial homeostasis ${ }^{3}$. 
Both mitochondrial and ER alterations could be reverted by a combination of sofosbuvir/daclatasvir, which are clinically approved direct-acting antivirals (DAAs) for the treatment of chronic HCV infection. In addition to providing structural insight into cellular aspects of HCV pathogenesis our study illustrates how cryo-SXT is a powerful three-dimensional wide-field imaging tool for the assessment and understanding of complex cellular processes in a setting of near native whole hydrated cells. Our results also constitute a proof of concept for the use of cryo-SXT as a platform that enables determining the potential impact of candidate compounds on the ultrastructure of the cell that may assist drug development at a preclinical level ${ }^{3}$.

\section{References:}

[1] Arslan, I, Tong, JR, Midgley, PA, Ultramicroscopy 106 (2006), p. 994.

[2] Carzaniga, R et al, Methods in cell biology 124 (2014), p.151.

[3] Pérez-Berná AJ et al, ACS Nano 10 (2016), p. 6597.

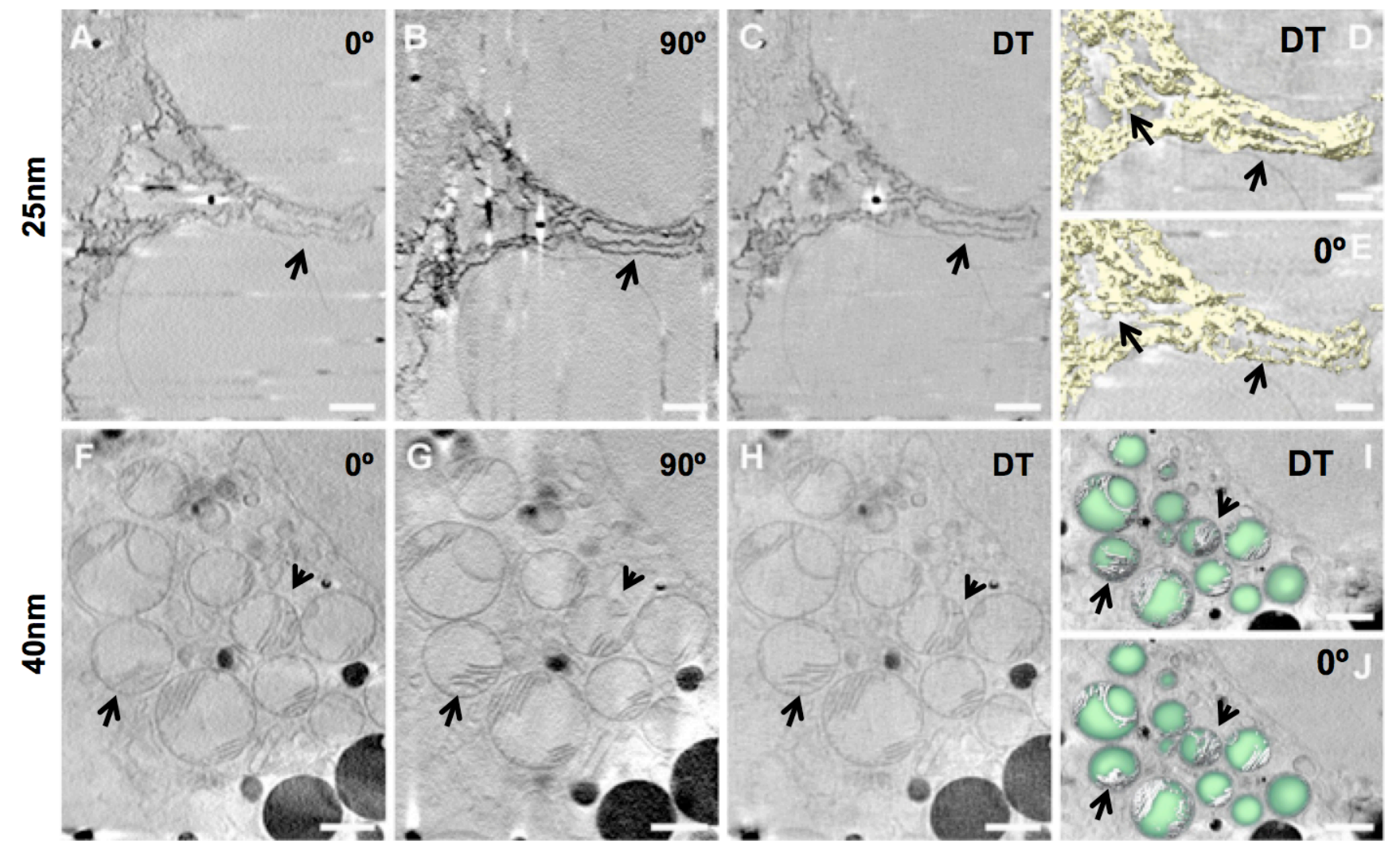

Figure 1. The fig shows the single tomograms (A \& F), the 90deg rotations ( $B$ \& $G$ ) and the dual tilt data combinations (C \& H) (Fig7, black arrows). Higher visibility of the features is clearly obtained in the DT combination as can be seen in (Fig7 D, E, I, J) 